\title{
Preoperative virtual simulation of adrenal tumors
}

\author{
Mikio Shiozawa, ${ }^{1}$ Naohiro Sata, ${ }^{1}$ Kazuyuki Endo, ${ }^{1}$ Masaru Koizumi, ${ }^{1}$ Yosikazu Yasuda, ${ }^{1}$ \\ Hideo Nagai, ${ }^{1}$ Hiroshi Takakusaki ${ }^{2}$ \\ ${ }^{1}$ Department of Surgery, Jichi Medical University, 3311 Yakushiji, Shimotsuke, Tochigi 329-0498, Japan \\ ${ }^{2}$ Department of Radiology, Jichi medical University, Shimotsuke, Tochigi, Japan
}

\begin{abstract}
Background: Retroperitoneal endoscopic adrenalectomy (EA) is recognized as a principal procedure for benign adrenal tumors. However, a limited visual field and a narrow working space make this approach difficult, particularly in cases of obese patients or small tumors. Using multidetector row CT (MD-CT), this study investigated the use of preoperative virtual simulation (PVS) to identify tumor and central vein locations for EA, and verified these findings during EA surgery.

Patients and methods: The study enrolled 11 cases comprising 10 adrenal adenomas and one ACTH-independent macronodular adrenal hyperplasia admitted to Jichi Medical University Hospital, Tochigi, Japan, between November 2003 and October 2006. Patients were evaluated in a lateral bending position using MDCT. 3D PVS images of ribs, vertebrae, kidneys, and adrenal tumors were generated and compared with real images obtained during EA.

Results: The PVS images clearly showed the relative locations of the adrenal tumor, kidney, and adjacent anatomical structures. These locations were verified during EA. The central vein was identified in the PVS images in all cases. Information derived from the PVS images assisted in the performance of EA surgery.

Conclusions: Preoperative 3D-simulation images using MD-CT contributed to the safety and efficiency of performing EAs.
\end{abstract}

Key words: Virtual simulation-Adrenal tumor-Endoscopic adrenalectomy-Retroperitoneal approach-Three dimension

Correspondence to: Mikio Shiozawa; email: shiozawa@jichi.ac.jp
Endoscopic adrenalectomy (EA) has become the standard treatment for benign adrenal tumors following the first report of endoscopic transperitoneal adrenalectomy for benign adrenal disease by Gagner et al. [1]. Transperitoneal and retroperitoneal approaches are the two principal surgical routes to the adrenal gland. While the transperitoneal approach is used more widely, the retroperitoneal approach offers particular advantages, although the narrower working space and fewer landmark organs make this latter technique more complicated [24]. Multidetector row CT (MD-CT) is a new type of CT that uses multiple detector rows, and provides finer image resolution and faster examinations than previous CT methodologies. MD-CT has provided new aspects for preoperative examinations in various fields [5-8], including adrenal tumor treatment [3,9]. Precise orientation in retroperitoneal EA is essential and reduces mortality and morbidity. The present study evaluated using MD-CT for preoperative virtual simulation (PVS) in the treatment of adrenal tumors. This technology was used to visually determine the relationship between the adrenal glands and adjacent organs, and the location of the central vein.

\section{Patients and methods}

From 1998 to 2006, 64 patients (31 males, 33 females; mean age: 50.1 years, ranging $18-77$ years) underwent EA in the Department of Surgery, Jichi Medical University Hospital, Tochigi, Japan. Over this period, the methodology applied to reach the adrenal gland gradually changed from a gasless transperitoneal route to a pneumoretroperitoneal method. From 2003, a retroperitoneal lateral approach became our standard method, and we introduced preoperative virtual simulation (PVS) using MD-CT. Recently, 11 cases ( 4 males, 7 females, mean age: 52.3 years, 5 with Cushing syndrome, 4 
with primary aldosteronism, 1 with pheochromocytoma, and 1 with hyperplasia) were evaluated using MD-CT and PVS. An MD-CT upper abdominal scan was performed with the patient in a lateral bending position, which was the same position as for the EA. All underwent scanning at $120 \mathrm{kV}$ independent of body mass index; 250-350 mAs was selected in relation with the body mass index. First scanning was performed without contrast medium, followed by administration of an intravenous bolus infusion of $100 \mathrm{~mL}(300-350 \mathrm{mgI} / \mathrm{mL}$ depending on the body weight) iodine contrast medium (Omnipaque; Daiichi Sankyo, Tokyo, Japan) at a rate of $3 \mathrm{~mL} / \mathrm{sec}$ using double-power injector. Second and third scanning was started at $30 \mathrm{~s}$ and $90 \mathrm{~s}$ after the beginning of the administration of the iodine contrast medium. The following parameters were used: detector setup, $0.75 \times 16 \mathrm{~mm}$; and helical pitch, 10.5/16 rows. Acquired $1.0-\mathrm{mm}$ slices were reformed by $1.0-\mathrm{mm}$ DICOM datasets. DICOM datasets were transferred to a workstation (M900 Quadra, Ziosoft) and PVS images generated. Adrenal tumors were first marked manually before postprocessing using the computer window levels. 3Dimages of ribs, vertebrae, kidney, and marked tumors were volume rendered. We also sought to mark the line of the central vein. The PVS images were evaluated in comparison to real images from the EA.

\section{A}

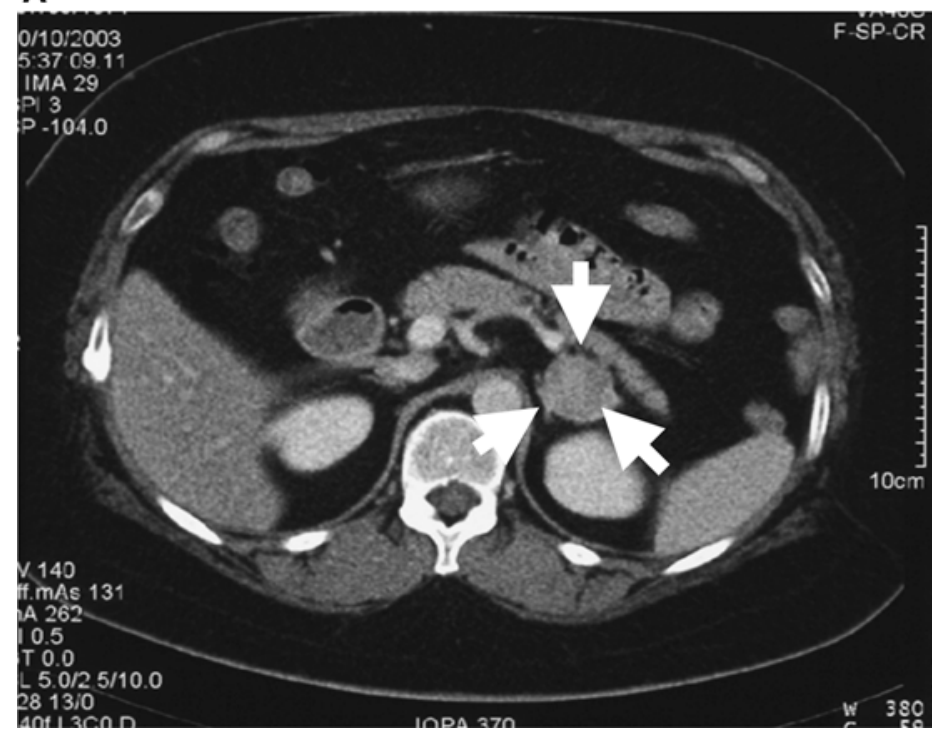

Fig. 1 Differing tumor position depending upon the patient position. Left adrenal adenoma (Cushing syndrome) $(3.0 \mathrm{~cm}$ ) (arrows in A-B) in a 32-year-old woman with central obesity.

\section{B}

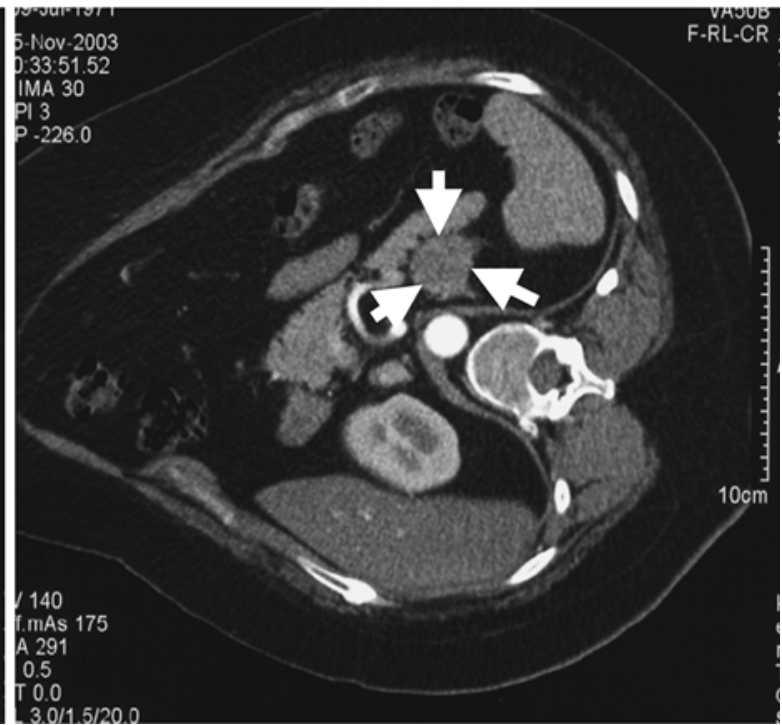

(A) CT in supine position. Note the tumor at the upper pole of the left kidney. (B) CT in right lateral bending position. Note the tumor on the head side.
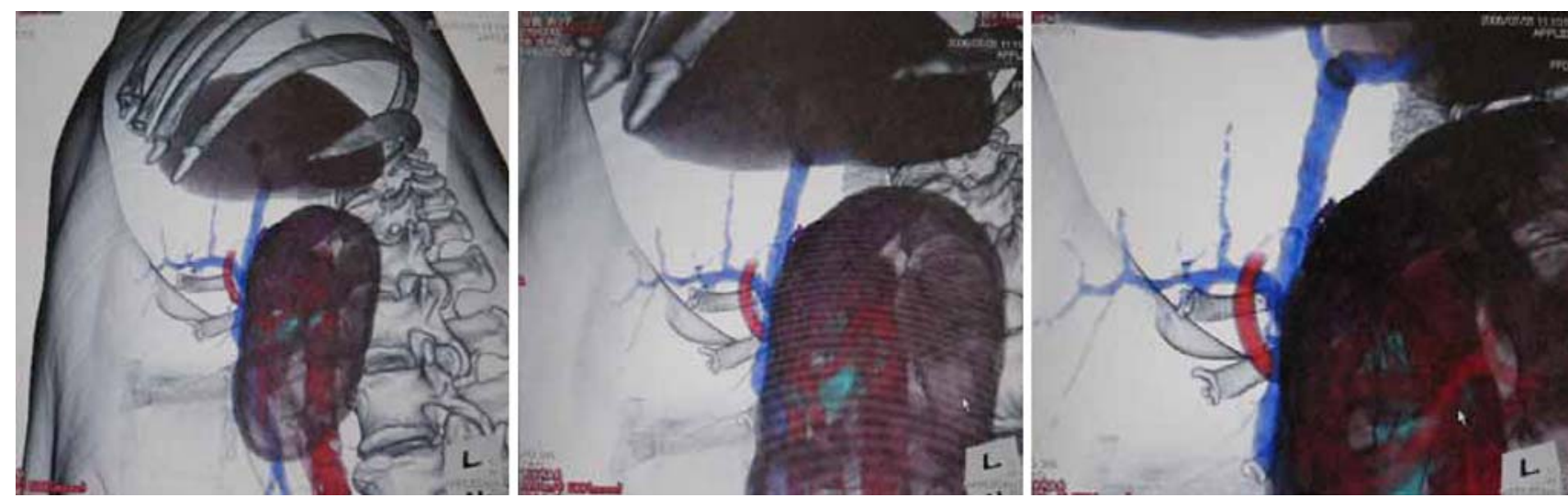

Fig. 2 Surgical approaches for endoscopic adrenalectomy. In a lateral bending position, a suitable video port position can be simulated to avoid the ,chasing sword' phenomenon. 
A

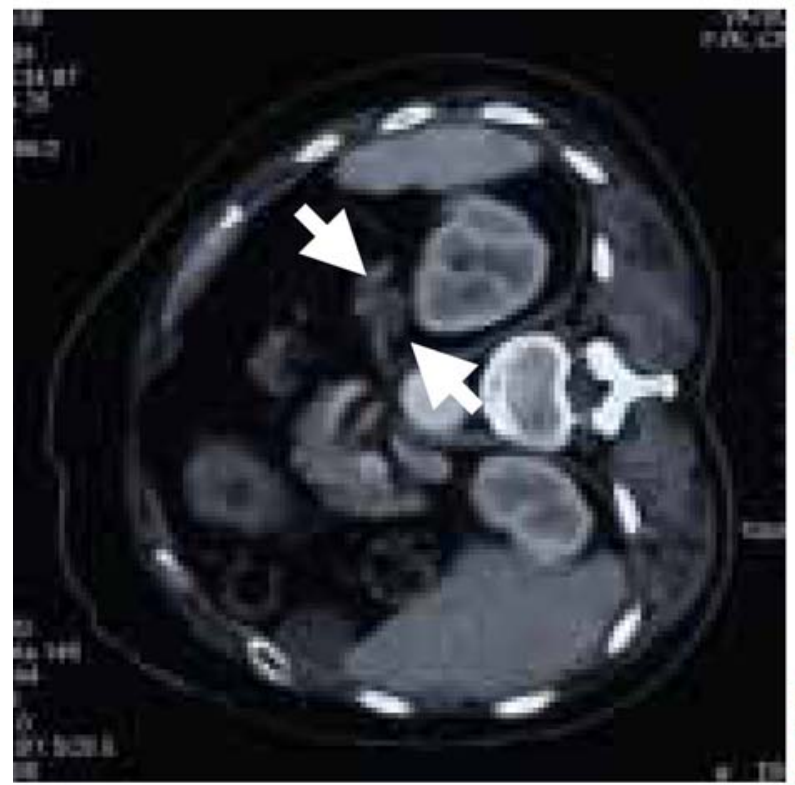

C

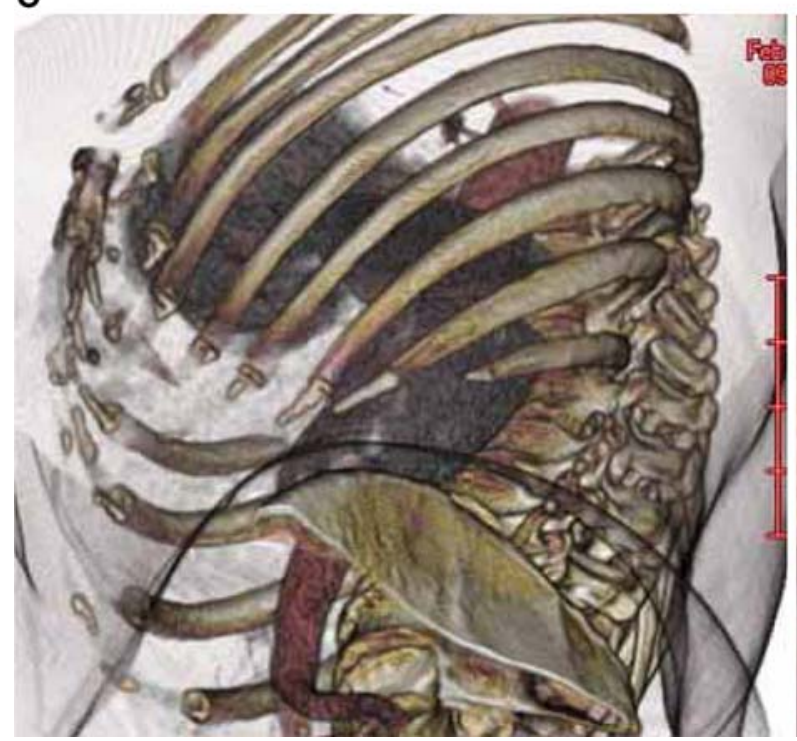

B

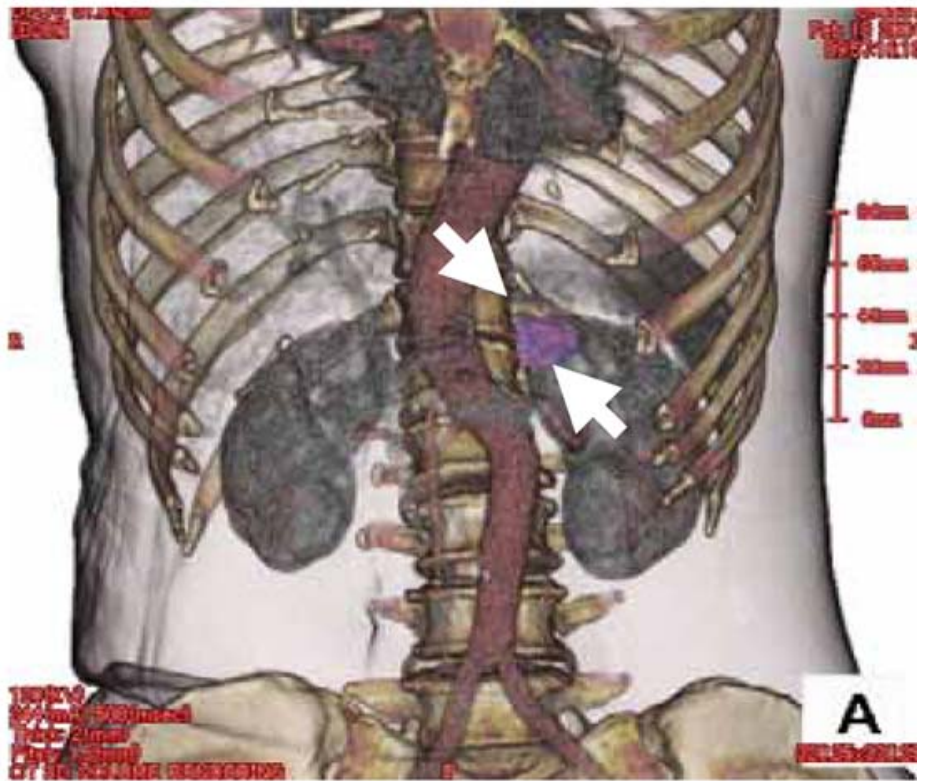

D

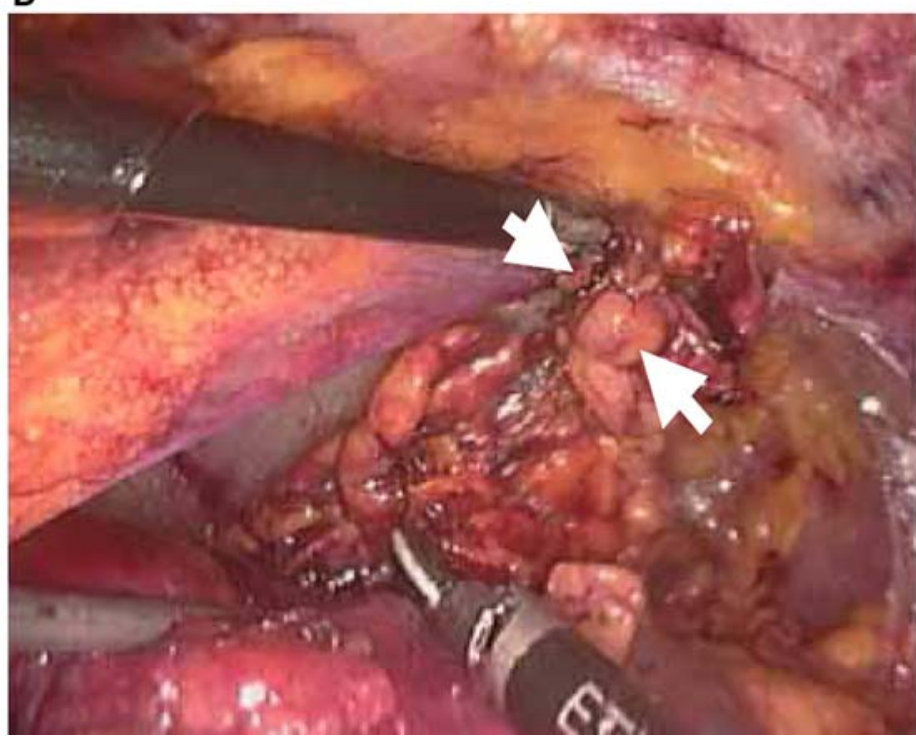

Fig. 3 Covered tumor. Left aldosterone-producing adenoma $(1.5 \mathrm{~cm})$ (arrows in A-B) in a 57-year-old woman. (A) Axial CT scan in the lateral position. (B) 3D-simulation CT of the front view. (C) The simulation view. (D) Real view during EA surgery. Note the left kidney completely hides the tumor in the simulated view. The upper pole of the kidney was mobilized and pushed to the psoas muscle.

\section{Results}

PVS images usually took approximately $30 \mathrm{~min}$ to be generated in each case. The MD-CT images differed depending upon whether the patient was in a lateral bending position or a supine position. The adrenal gland was longitudinally shifted to the upper side in the lateral bending position, especially in patients who had significant fat deposits (Fig. 1).

We simulated the port position using PVS images. PVS images of the adrenal tumor, kidney, ribs, vertebrae, and pelvis were generated and rotated longitudinally to fix the rational direction of the endoscopic port, which would be inserted in the mid-axillary line and $1 \mathrm{~cm}$ below the tip of the twelfth rib (Fig. 2). The location of the adrenal tumor was then evaluated in relation to the kidney and was classified as either covered or uncovered. Covered meant the adrenal tumor was completely hidden in terms of the virtual endoscopic port position (Fig. 3c), while uncovered meant it was not completely hidden (Fig. 4c). Of the 6 left-sided tumors, 2 were classified as uncovered and 4 covered. All 5 right-sided tumors were uncovered. In all cases, these classifications were verified 

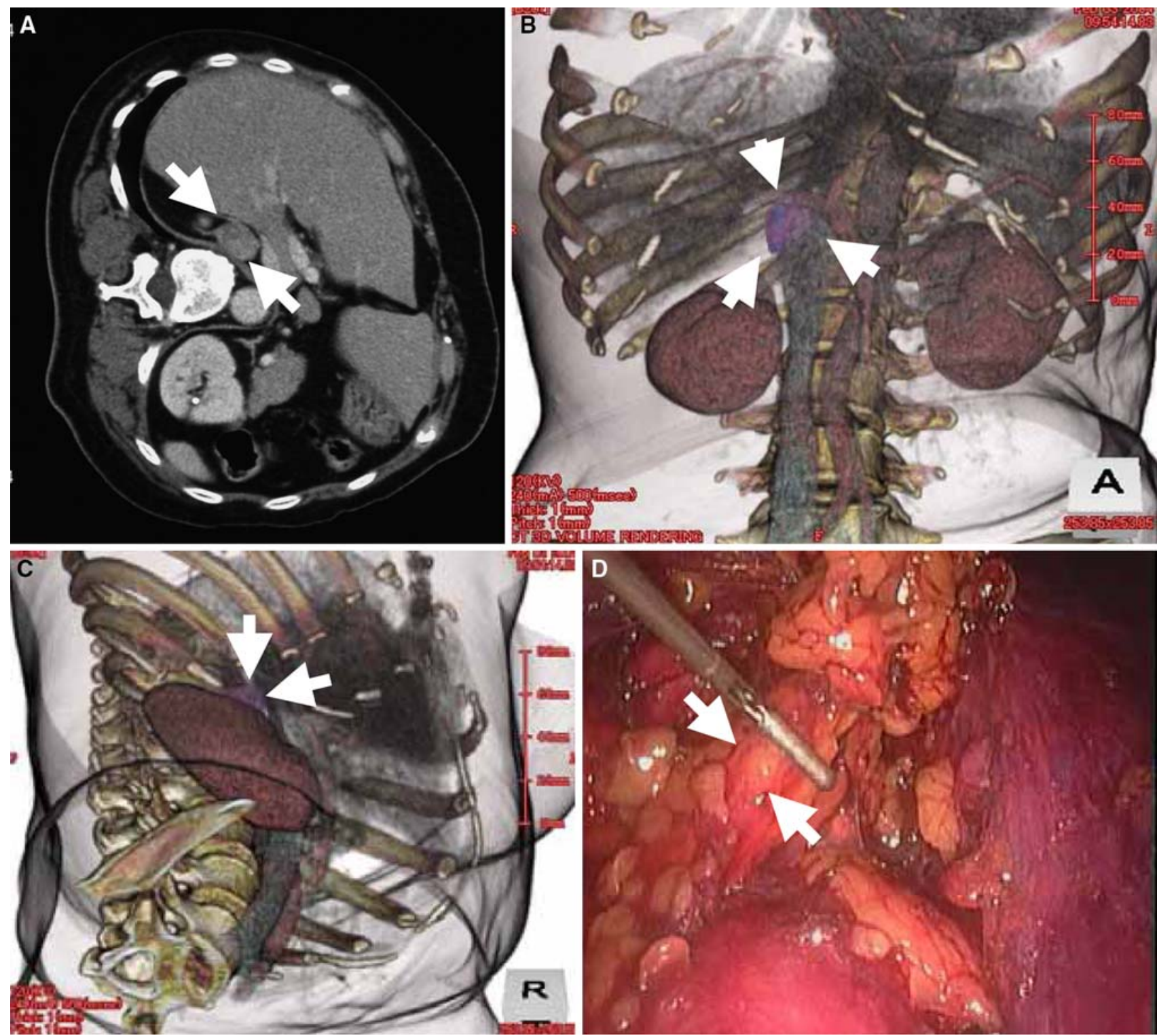

Fig. 4 Uncovered tumor. Right adrenal adenoma (Cushing syndrome) $(1.5 \mathrm{~cm})$ (arrows in $\mathbf{A}-\mathbf{B})$ in a 55-year-old woman. (A) Axial CT scan in the lateral position. (B) 3D-simulation CT of the front view. (C) The simulated view. (D) Real view during

EA surgery. Note the tumor is not obscured by the left kidney in the simulated view. The adrenal gland was easily revealed without mobilizing the kidney.

using real images from endoscopic surgery (Fig. 3d, 4d). Covered tumors were not visible without significantly mobilizing the kidney during surgery (Fig. 3d). The PVS images were found to assist in the undertaking of the EA.

Determining the locations of the right and left central veins is critical in EA. The right central vein is short and directly flows into the inferior vena cava, and the left central vein is long and flows into the left renal vein. From February 2006, we used PVS images to identify the central vein in 6 cases. This procedure was successful on all 6 occasions (Figs. 5, 6). The PVS findings were verified by the real findings during endoscopic surgery (Figs. 5d, 6d).

Endoscopic adrenal-preserving surgery was performed in 6 of the 11 cases. Such surgery requires knowledge of the precise location of the tumor on the gland. PVS images successfully showed the relative location of the tumor in the normal gland and the location of the central vein (Fig. 7). PVS images assisted in the undertaking of safe adrenal-preserving surgery.

\section{Discussion}

The transperitoneal approach for adrenalectomies is common because it offers easy orientation, dissection, and mobilization in the abdominal cavity. However, this 

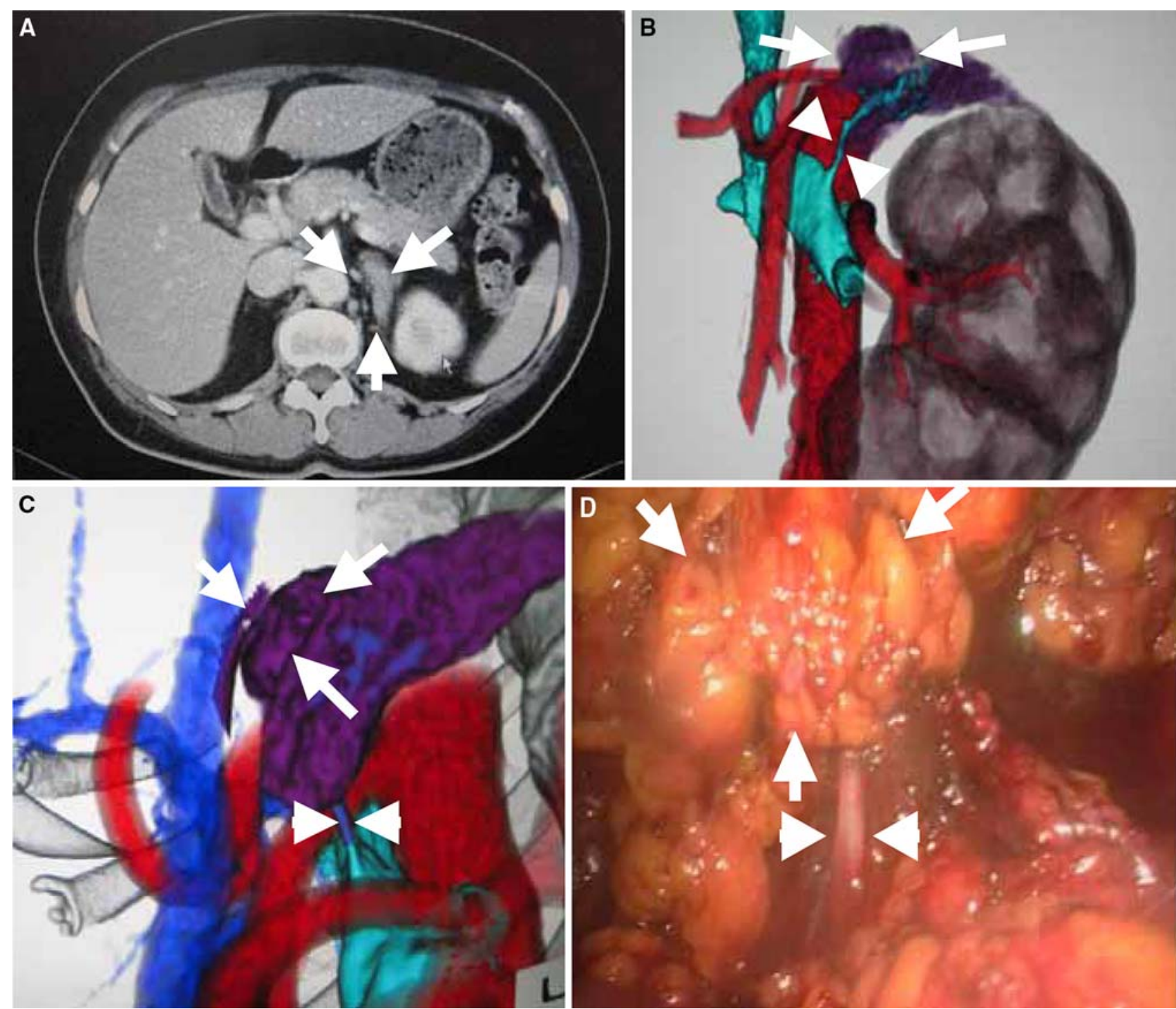

Fig. 5 Left central vein. Left aldosterone-producing adenoma $(3.0 \mathrm{~cm})$ (arrows in A-D) in a 58-year-old woman The left main adrenal vein exists in the inferior aspect of the adrenal gland to drain into the left renal vein (short arrows in B-D).

procedure has potential disadvantages including adhesion formation, postoperative bowel obstruction, and intraoperative injury to adjacent organs. The most practical advantage of the retroperitoneal approach is avoiding possible injury to abdominal organs [2]. The procedure is more complicated than the transperitoneal approach because of fewer anatomic landmarks, abundant retroperitoneal adipose tissue, and a smaller surgical working space [2-4].

Recently, CT and MRI have been used for surgical planning of procedures such as laparoscopic donor nephrectomy [5], laparoscopic resection of gastric cancer [10], and living related liver donor resection [11]. Using cut-plane imaging without three-dimensional (3D) reconstruction, Hurley et al. were the first to simulate the relationship between adrenal masses and adjacent organs using 3D volume-rendered helical CT [3], and evaluate the interaction of adrenal masses with the diaphragm, liver, spleen, and kidneys. In the present study, we introduced a new 3D-image reconstruction technique, PVS, as a simulating tool for EAs which is more precise than previous methods. We found that PVS images provided realistic virtual views and precise tumor locations. PVS highlighted that the adrenal gland position differed according to the patient position. This finding is particularly pertinent given most patients are assessed by CT in a supine position, yet undergo the EA in a lateral bending position.

A retroperitoneal EA approach is especially challenging in obese patients. Cushing syndrome patients are candidates for EA, and are frequently obese and have fragile connective tissue. It is important to know the relationship between the adrenal gland and the kidney as landmark organ. We found that PVS images created 

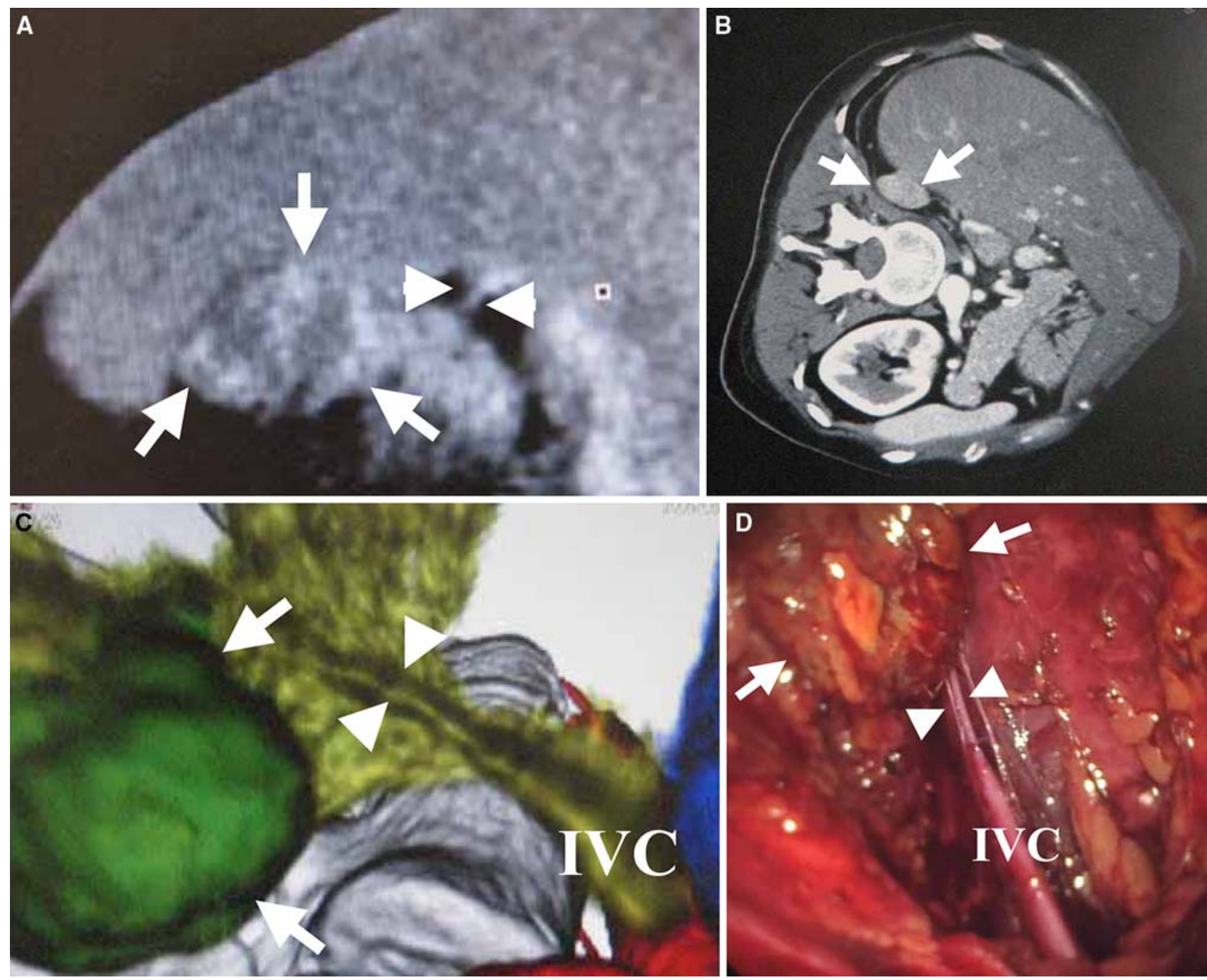

Fig. 6 Right central vein. Right adrenal adenoma (Cushing syndrome) $(2.6 \mathrm{~cm})$ (arrows in A-B) in a 31-year-old woman. (A) Coronal scan. (B) Axial scan in the lateral position. (C) The simulated view. (D) Real view during EA surgery. The

from MD-CT in the lateral bending position provided "real" virtual images even in Cushing syndrome cases. In the present study, we classified adrenal tumors as either covered or uncovered depending on the relative locations of the kidney and the adrenal gland. In all cases these classifications were verified during EAs, and PVS was found to provide rapid and useful information for EAs. The present study found that all right-sided tumors were of the covered type and were found after adequately mobilizing the right kidney, while left tumors were generally found more easily.

The final step in EA is identification and dissection of the central vein. This step is especially crucial in pheochromocytoma to avoid excessive release of catecholamines $[2,12,13]$. Hurley et al. identified the central vein using volume-rendered CT scan images in $63 \%$ of cases simulated view shows that the short central vein (short arrows in $\mathbf{A}$ and $\mathbf{C}$ ) drains into the IVC, and this was confirmed during surgery (short arrows in D).

(77\% of left-sided cases and 38\% of right-sided cases). Scatarige et al. investigated the left adrenal vein in a group of living renal donors before laparoscopic nephrectomy and were able to identify the left central vein in $92.5 \%$ of cases [14]. In the present study, visualization of the arterial and venous anatomy and the location of central vein was achieved using PVS in all 6 attempted cases.

Adrenal-preserving adrenalectomy has been reported in the resection of adrenal adenomas [4, 15-17]. We found that PVS images provided useful information for this procedure, such as the relative location of the tumor and central vein, and the location of arteries. We were able to make a precise plan of the surgical margin and the amount of residual adrenal gland using PVS images. 
A

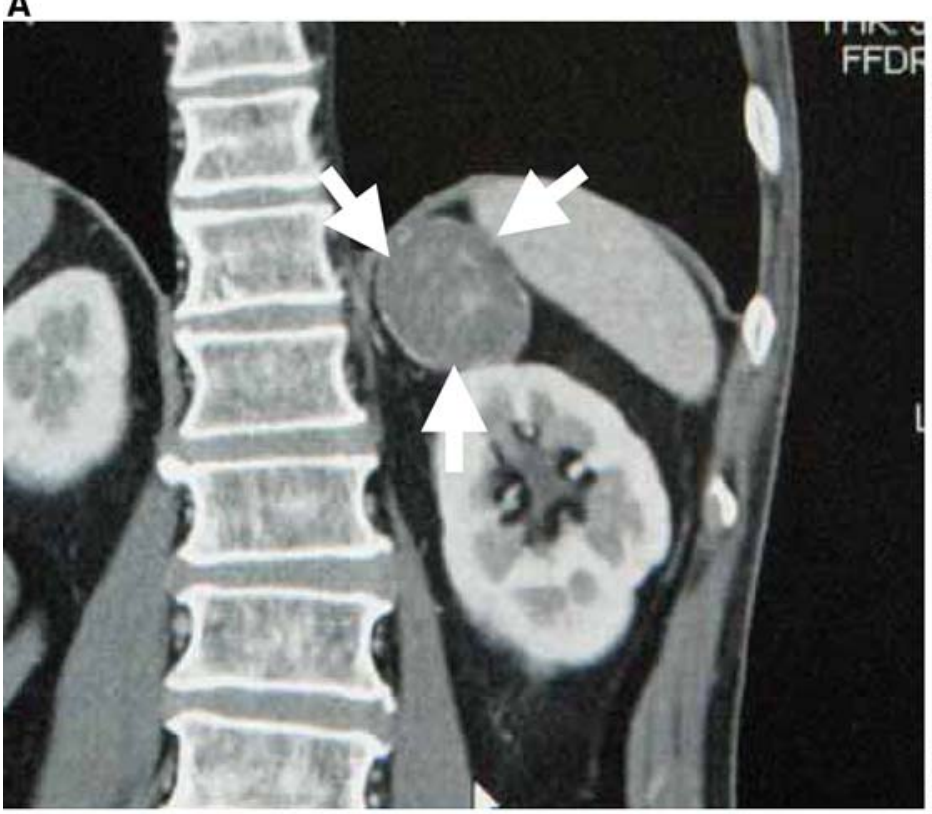

C

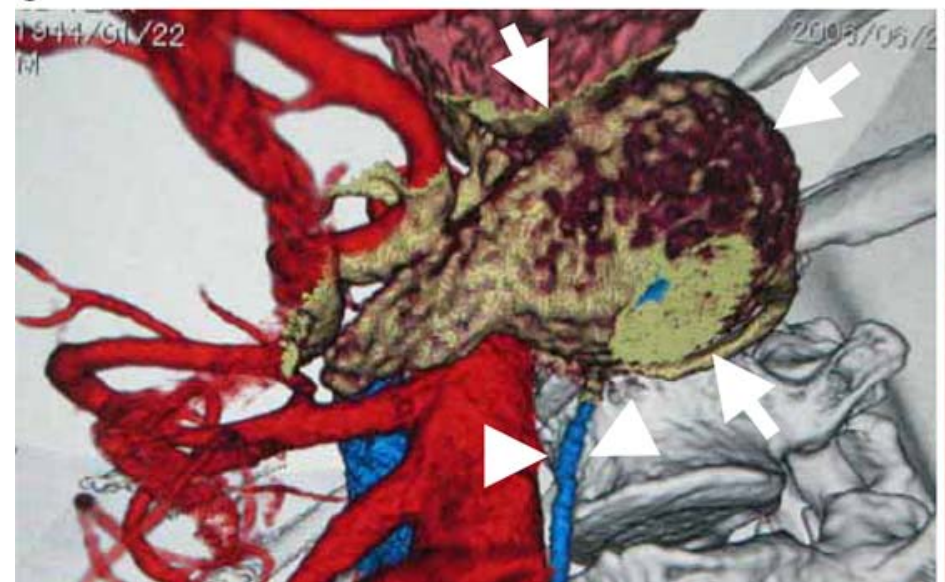

B

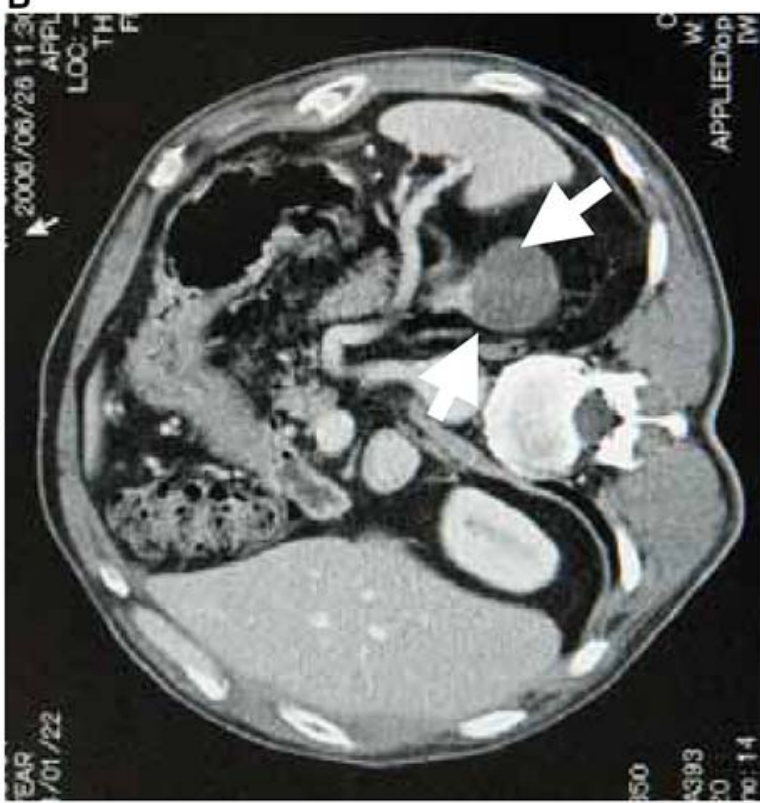

D

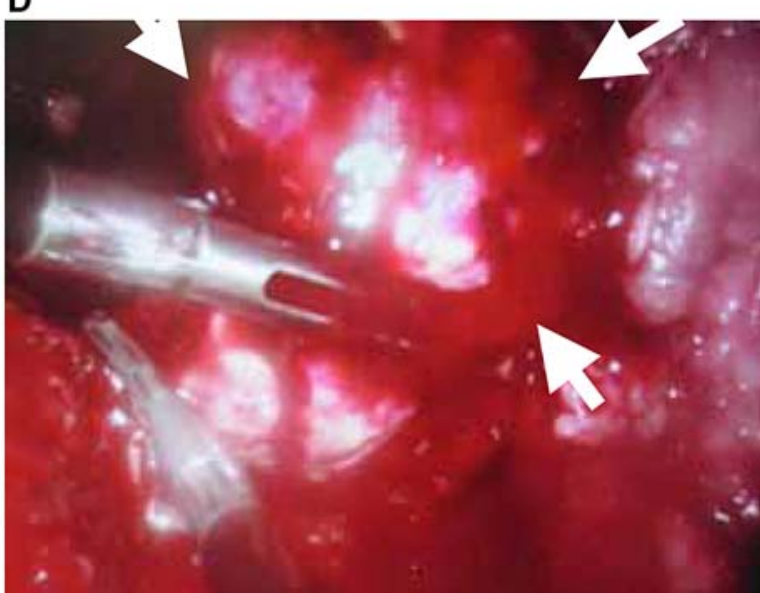

Fig. 7 Endoscopic adrenal-preserving surgery. Left adrenal adenoma (Cushing syndrome) $(4.0 \mathrm{~cm}$ ) (arrows in a-d) in a 62-year-old man. (A) Coronal scan. (B) Axial scan in the lateral position. (C) The simulated view. (D) Real view during EA

We conclude that PVS images can be easily and quickly generated using MD-CT. These images provide sufficient information to perform EAs.

Open Access. This article is distributed under the terms of the Creative Commons Attribution Noncommercial License which permits any noncommercial use, distribution, and reproduction in any medium, provided the original author(s) and source are credited.

\section{References}

1. Gagner M, Lacroix A, Bolte E (1992) Laparoscopic adrenalectomy in Cushing's syndrome and pheochromocytoma. N Engl J Med 327:1033

2. Munver R, Ilbeigi P (2005) Retroperitoneal laparoscopic adrenalectomy. Curr Urol Rep 6:72-77

3. Hurley ME, Herts BR, Remer EM, et al. (2003) Three-dimensional volume-rendered helical CT before laparoscopic adrenalectomy. Radiology 229:581-586 surgery. The simulated view shows the adenoma location in the adrenal gland and the short central vein (short arrows in C) more precisely than the axial view. Endoscopic adrenalpreserving surgery was performed (D).

4. Micali S, Peluso G, De Stefani S, et al. (2005) Laparoscopic adrenal surgery: new frontiers. J Endourol 19:272-278

5. Smith PA, Ratner LE, Lynch FC, et al. (1998) Role of CT angiography in the preoperative evaluation for laparoscopic nephrectomy. RadioGraphics 18:589-601

6. Kikuchi S, Futawatari N, Sakuramoto S, et al. (2006) Pre-operative tumor assessment of patients with gastric cancer based on virtual endoscopy using multidetector-row computer tomography. Anticancer Res 26:4641-4645

7. Sata N, Endo K, Shimura K, et al. (2006) A new 3-D diagnosis strategy for duodenal malignant lesions using multidetector row CT, CT virtual duodenoscopy, duodenography, and 3-D multicholangiography. Abdom Imaging Epub ahead of print

8. Kamiyama T, Nakagawa T, Nakanishi K, et al. (2006) Preoperative evaluation of hepatic vasculature by three-dimensional computed tomography in patients undergoing hepatectomy. World J Surg 30:400-409

9. Mitterberger M, Pinggera GM, Peschel R, et al. (2006) The use of three-dimensional computed tomography for assessing patients before laparoscopic adrenal-sparing surgery. BJU Int 98:1068-1073 
10. Lee SW, Shinohara H, Matsuki M, et al. (2003) Preoperative simulation of vascular anatomy by three-dimensional computed tomography imaging in laparoscopic gastric cancer surgery. J Am Coll Surg 197:927-936

11. Bogetti JD, Herts BR, Sands MJ, et al. (2001) Accuracy and utility of 3-dimensional computed tomography in evaluating donors for adult living related liver transplants. Liver Transpl 7:687-692

12. Guazzoni G, Cestari A, Montorsi F, et al. (2004) Laparoscopic treatment of adrenal diseases: 10 years on. BJU Int 93:221-227

13. Cestari A, Naspro R, Rigatti P, et al. (2005) Laparoscopic adrenalectomy and adrenal-preserving surgery. Curr Opin Urol 15:6974
14. Scatarige JC, Horton KM, Ratner LE, et al. (2001) Left adrenal vein localization by $3 \mathrm{D}$ real-time volume-rendering CTA before laparoscopic nephrectomy in living renal donors. Abdom Imaging 26:553-556

15. Jeschke K, Janetschek G, Peschel R, et al. (2003) Laparoscopic partial adrenalectomy in patients with aldosterone-producing adenomas: indications, technique, and results. Urology 61:69-72

16. Ishidoya S, Ito A, Sakai K, et al. (2005) Laparoscopic partial versus total adrenalectomy for aldosterone producing adenoma. J Urol $174: 40-43$

17. Iihara M, Suzuki R, Kawamata A, et al. (2003) Adrenal-preserving laparoscopic surgery in selected patients with bilateral adrenal tumors. Surgery 134:1066-1072 